\section{${ }^{1}$ Sakarya Üniversitesi Eğitim ve Araştırma Hastanesi, Biyokimya, Sakarya, Türkiye e-posta: drmozdin33@gmail.com}

Atıf için: Mehmet ÖZDiN, Tip II Diabetes Mellituslu Hastalarda Retinopati ile HbA1c Arasındaki Iliş̧ki, Balıkesir Medical Journal, 2020;4(2): 41-45

Başvuru Tarihi: 08.05.2020

Kabul Tarihi: 16.06 .2020 Yayınlanma Tarihi: 23.06.2020

Sorumlu Yazar:

Uğur Ergün,

Balıkesir Üniversitesi Tıp

Fakültesi iç Hastalıkları Anabilim Dalı, Balıkesir, Türkiye

e-posta:

mdbalkes10@gmail.com

\title{
Tip II Diabetes Mellituslu Hastalarda Retinopati ile HbA1c Arasındaki İlişki
}

\author{
The Relationship Between Retinopathy and HbA1c in Type II Diabetes \\ Mellitus Patients
}

Mehmet ÖZDiN ${ }^{1}$

Öz

Amaç: Bu çalışmada amacımız; Sakarya Üniversitesi Eğitim ve Araştırma Hastanesi (SÜEAH) göz hastalıkları polikliniğine müracaat eden Tip II Diabetes Mellitus (Tip II DM) hastalarında Retinopati görülme sıklığı ile HbA1c düzeyleri arasında muhtemel ilişkinin incelenmesidir.

Gereç ve Yöntemler: Çalışmanın hasta popülasyonu SÜEAH göz hastalıkları polikliniğine müracaat eden ve DR tanıSı konulan kişilerden oluşturuldu. Retrospektif olarak yapılan çalışmanın hasta verileri hastane bilgi yönetimi sistemi (HBYS) üzerinden elde edildi. Dahil edilme kriterlerimize uyan eden 38 DR'li hastanın dosya verileri incelendi ve HbA1c düzeyleri tespit edildi. Hipertansiyon başta olmak üzere kardiyovasküler hastalığı olanlar ile primer veya sekonder kronik göz hastalığı olanlar çalışmaya dahil edilmemiştir. Elde edilen bulgular kontrol grubumuzla karşılaştırıldı. Çalışmaya 22 bayan, 16 erkek olmak üzere toplam 38 hasta dahil edildi. Çalışmaya alınan hastaların ortalama yaşları $61.32 \pm 9.87$ olarak tespit edildi. Kontrol grubumuz 25 bayan, 13 erkek olmak üzere 38 kişiden oluşturuldu. Kontrol grubumuz ve ortalama yaşları $58.32 \pm 11.97$ olarak tespit edildi.

Bulgular: Toplamda 38 DR hastası değerlendirildi. Hastanemizin göz hastalıkları polikliniğine müracaat eden DR hastaların $\mathrm{HbA} 1 \mathrm{C}$ düzeyleri $\% 9.05 \pm 1.72$, kontrol grubunun $\mathrm{HbA} 1 \mathrm{C}$ düzeyleri ise $\% 4,97 \pm 0.29$ olarak tespit edildi. DR hastaların $\mathrm{HbA} 1 \mathrm{C}$ düzeyleri ile kontrol grubunun HbA1C düzeyleri arasında istatiksel olarak anlamlı bir fark olduğu görüldü ( $p<0.05$ ). DR hastaların $\mathrm{HbA} 1 \mathrm{C}$ düzeyleri ile kontrol grubunun $\mathrm{HbA1C}$ düzeyleri arasında da negatif bir korelasyon varlığı ( $r=-0,21$ ) saptandı.

Sonuç: Çalışmamızda DR'li hastalarda HbA1c değerlerinin kontrol grubuna göre yüksek olduğu tespit edildi. Bu sonuç, tip II DM hastalarında glukoz düzeylerinin regüle edilmesinin ve HbA1c takiplerinin iyi yapılmasının önemini bir kez daha ortaya çıkardı.

Anahtar Kelimeler: Tip II Diabetes mellitus; diabetik retinopati; HbA1c.

\section{ABSTRACT}

Aim: Our aim in this study is to examine the possible relationship between the frequency of occurrence of Retinopathy in patients, who applied to the ophthalmology outpatient clinic of Sakarya University Training and Research Hospital (SUTRH), with Type II Diabetes Mellitus (Type II DM) and HbA1c levels.

Materials and Methods: The patient population of the study was composed of people who applied to the SUTRH ophthalmology outpatient clinic and were diagnosed with DR. Patient data of the study, which was conducted retrospectively, were obtained through the hospital information management system (HIMS). The file data of 38 DR patients who met our inclusion criteria were analyzed and HbA1c levels were determined. Those with cardiovascular disease, primarily those with hypertension, and primary or secondary chronic eye disease were not included in the study. The findings were compared with our control group. A total of 38 patients, 22 females and 16 males, were included in the study. The mean age of the patients included in the study was $61.32 \pm 9.87$ years. Our control group consisted of 38 individuals ( 25 females, 13 males). The mean age of the control group was $58.32 \pm 11.97$ years.

Results: A total of 38 DR patients were evaluated. HbA1C levels of the DR patients who applied to our ophthalmology outpatient clinic were $9.023 \pm 1.72 \%$ and $\mathrm{HbA} 1 \mathrm{C}$ levels of the control group were $9.023 \pm 1.72 \%$. $\mathrm{HbA1C}$ levels of the DR patients and $\mathrm{HbA} 1 \mathrm{C}$ levels of the control group were statistically significant $(p<0.05)$. There was a positive cor relation $(r=$ 0.511 ) between $\mathrm{HbA} 1 \mathrm{C}$ levels of $\mathrm{DR}$ patients and $\mathrm{HbA} 1 \mathrm{C}$ levels of the control group.

Conclusion: In our study, HbA1c values were found to be higher in patients with DR than in the control group. This result once again demonstrated the importance of regulating glucose levels and keeping HbA1c well monitored in type II DM patients.

Keywords: Type II Diabetes mellitus; diabetic retinopathy; HbA1c. 
Tip II DM, insülin hormonunun noksanlığı veya etki gösterememesi sonucu ortaya çıkmaktadır. Başta karbonhidratlar olmak üzere, yağ ve proteinlerden vücudun yeterince faydalanamadığı hiperglisemi ile karakterize kronik seyirli bir hastalıktır (1). DM son yıllarda hızla artış gösteren, ciddi komplikasyonlara yol açan bir sağlık problemidir (2). Tüm dünyada ve Türkiye'de DM görülme sıklığı \%10'un üzerindedir. Bu olguların \%9095'ini tip II DM oluşturur iken, kalan \%5-10'unu tip I DM ve \%2-3'ünü ise diğer diyabet formları oluşturur (1). Tip II DM; özellikle küçük kan damarlarını tutan sıklıkla gözlerde ve böbrekte irrevezibil dejenerasyona neden olan karmaşık bir metabolik hastalıktır (3-5). Tip II DM erişkinlerde gözde görülen en önemli komplikasyonu körlüktür $(6,7)$. Tip II DM hastalarında gözlenen asıl problem gözün kapiller vasküler yapısındaki tahribat ve dolayısı ile retinanın beslenmesinde görülen azalmadır temel sorunu gözün kapiller vasküler yapısında hasar oluşması ve retinanın beslenememesidir. Tip II DM hastaların yaklaşık \%2'sinde DR'ye bağlı körlük olduğu bilinmektedir $(8,9)$. Tip II DM hastalarında glokom, katarakt ve gözün diğer bozuklukları daha erken dönemlerde ortaya çıkmaktadır $(6,10)$. İnsülinin tip II DM tedavisinde kullanılmaya başlamasıyla bu hastalarda gözlenen yaşam sürelerinde artış kaydedilmektedir. Hastaların yaşam süresindeki bu artıştan dolayı tip II DM bağlı olarak görülen komplikasyonlara rastlama sıklığını artırmıştır. DM tanı yöntemlerindeki gelişmeler bu hastalığın teşhisinin erken konulmasına neden olmuştur. Glikozillenmiş hemoglobin tayini yöntemi ile ölçülen HbA1c ölçümleri de DM tanısında önemli yer almaktadır (11). Bu çalışmada tip II DM'lu hastalarda diabetin mikrovasküler komplikasyonlarından olan DR ile HbAlc arasındaki ilişki araştırılmıştır.

\section{GEREÇ VE YÖNTEMLER}

Çalışmamız hastanemizin göz hastalıkları polikliniğine müracaat eden DR hastalarından oluşmuştur. Müracaat eden 38 DR'li hastalarının dosya verileri incelenerek HbA1c düzeyleri tespit edilmiştir. Hipertansiyon başta olmak üzere kardiyovasküler hastalığı olanlar ile primer veya sokonder kronik göz hastalığı olanlar çalışmaya dahil edilmemiştir. Çalışmaya 22 bayan, 16 erkek olmak üzere toplam 38 hasta dahil edilmiştir. Çalışmaya alınan hastaların ortalama yaşları 61.32 \pm 9.87 dir. Kontrol grubumuz 25 bayan, 13 erkek olmak üzere 38 kişiden oluşmuştur. Kontrol grubumuz ve ortalama yaşları $58.32 \pm 11.97$ dir. Verilerin istatistiksel değerlendirilmesi için SPSS for Windows 22.00 paket programı kullanıldı. Parametrik veriler ortalama \pm standart sapma (SS) ile, nonparametrik veriler \% ile gösterildi. Etik Komite Onayı: Bu çalışmada, Helsinki protokolü komitesinin gereklerine uygun olarak yapıldı ve Sakarya Üniversitesi Kurumsal Etik Komitesi tarafından onaylandı (Karar no:06/11/2019-E.13958). HbA1C testleri ADAMS, A1C, HA-8180V cihazında çalışılmıştır.

Biyokimyasal test parametresinin performans özellikleri:

HbA1c'nin ölçüm metodunda ters faz katyon değişin kromotografisi kullanarak ölçer. Örnek yüksek performanslı sıvı kromotografisi (HPLC) yöntemi ile çeşitli hemoglonin bileşenlerine ayrıştırılarak ölçüm tamamlanır. Yaygın kullanılma sebepleri arasında duyarlılığı, kantitatif tayinlere kolaylıkla uyarlanabilir olmasıdır. HPLC yönteminin spesifitesi ve sensitivitesi yüksek olup $\mathrm{HbS}$ ve $\mathrm{HbC}$ gibi anormal hemoglobinleri de ayırt etmeyi sağlamıştır. 


\section{BULGULAR}

Çalışmamızda hastanemizin göz hastalıkları polikliniğine müracaat eden toplam 38 DR'si olan hasta değerlendirildi. Hastanemizin göz hastalıkları polikliniğine müracaat eden DR hastaların HbA1C düzeyleri $\% 9.05 \pm 1.72$, kontrol grubunun HbA1C düzeyleri \%4,97 \pm 0.29 tespit edildi. DR hastaların HbA1C düzeyleri ile kontrol grubunun $\mathrm{HbA} 1 \mathrm{C}$ düzeyleri arasında istatiksel olarak anlamlı olduğu $(p<0.05)$ tespit edildi. DR hastaların HbA1C düzeyleri ile kontrol grubunun HbA1C düzeyleri arasında da negatif bir korelasyon varlığı $(r=-0,21)$ saptandı (tablo 1) (grafik 1).

\begin{tabular}{|l|c|c|c|c|c|}
\hline & $\mathrm{n}$ & yaş & HbA1c & $\mathrm{p}$ & $\mathrm{r}$ \\
\hline Hasta & 38 & $61.32 \pm 9.87$ & $9.05 \pm 1.72$ & $\mathrm{p}<0.05$ & $-0,21$ \\
\hline Kontrol & 38 & $58.32 \pm 11.97$ & $4.97 \pm 0.29$ & $\mathrm{p}<0.05$ & $-0,21$ \\
\hline
\end{tabular}

Tablo 1. Hasta ve Kontrol Grubuna ait veriler

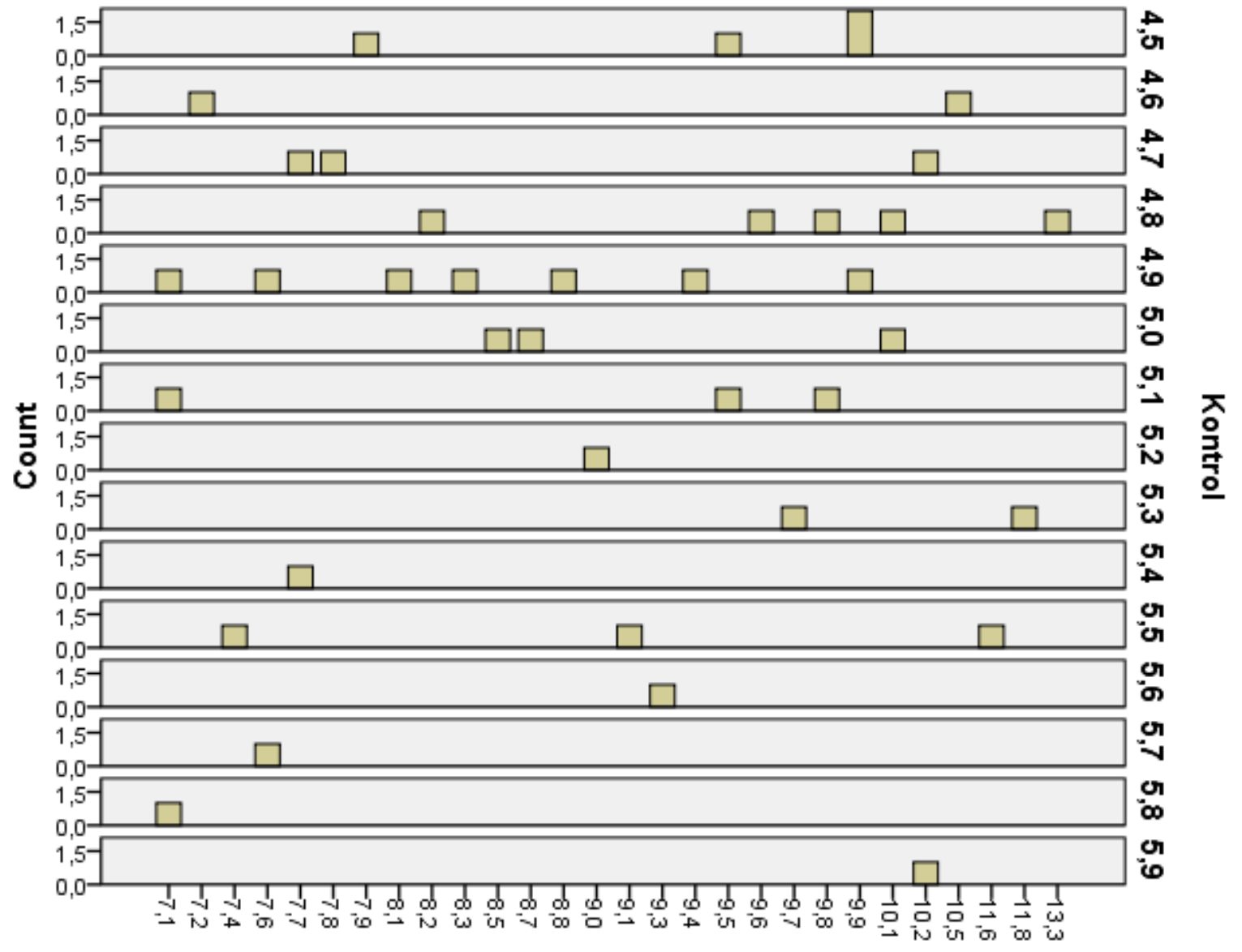

Hasta

Grafik 1. Diabetik Retinopatili hastalar ile kontrol grubundaki HbA1c dağılımı 


\section{TARTIŞMA}

HbA1c tip II DM tanısında ve kan şekeri regülasyonunun izlenmesinde yol gösterici bir parametredir. HbA1c düzeyleri tip II DM'de kan glukozu regülasyonunun iyi veya kötü olduğu tespitinde de faydalı olup, bu hastalığın komplikasyonları ile yakından ilişkilidir (11). Ferrer ve arkadaşları (12), her iki cinsten 40-65 yaşları arasındaki 140 hastayı (\%44 Tip I DM, \%56 Tip II DM) 5 yıllık sürede takip etmişlerdir. Bu hastalarda mikrovasküler komplikasyonlar açısından değerlendirmişler. Olguları HbA1c düzeyleri \%8'den düşük ve yüksek olmak üzere iki gruba ayırmışlardır. Yaptıkları bu çalışmada özellikle Tip II DM'lu hastalarda DR sıklığı HbA1c düzeyi \%8'den fazla olanlarda anlamlı olarak yüksek bulunmuştur. Agardh ve arkadaşları (13), iki yıl boyunca 1362 olguyu (389 Tip I DM, 973 Tip II DM) izlemişlerdir. DR'si olmayanların ortalama HbAlc düzeyleri \%8,1 iken, DR'si olanların ortalama HbAlc düzeyleri \%8,6 olarak saptanmıştır. Yine Agardh ve arkadaşlarının (13) takip ettiği tip II DM'lu hastalarda DR görülmeyen hastaların ortalama diyabet süresi 11,5 yıldır. DR'si olanlarda ise bu süre ortalama olarak 12,6 yıl olarak tespit edilmiştir. Ülkemizde yapılan diğer bir çalışmada tip II DM olanlar izlenmiştir. 5 yıldan az süreli tip II DM hastalarda DR sıklığı \%21, 6-10 yıllık tip II DM olan hastalarda ise \%29,8 olarak bulmuşlardır. 11-15 yıllık tip II DM hastası olanlarda \%42 iken, 16 yıl ve daha fazla süredir tip II DM hastası olanlarda ise sıklık \%46,5 olarak saptanmıştır (14). Tip II DM'lu hastalarda HbA1c değeri olan \%7'ye ulaşmanın mikrovasküler ve makrovasküler komplikasyonları azaltmadaki önemi gösterilmiştir (15). Diğer bir çalışmada $\mathrm{HbA} 1 \mathrm{C}$ değerleri retinopati olan hastalarda \%7.4 ile \%7.9 arasında iken retinopati olmayan hastalarda ise \%6.8-\%7.0 arasında tespit edilmiştir (16). Yapılan bir çalışmada ise HbA1C değerinin \%1 düşmesinin DR gelişimini \%37 oranında az görülmesine neden olmuştur. Bu nedenle de HbA1C değerinin \%7'nin altında olması tavsiye edilmiştir (18). Yapılan bir diğer çalışmada DR'si olan hasta grubunda HbA1C ortalaması \%8,7 iken, DR'si olmayan hasta grubunda ise \%8,0 olarak tespit edilmiştir (19). Yaptığımız çalışmada tip II DM hastası olup DR'si olanlar ile kontrol grubunu karşılaştırdığımızda istatistiki yönden anlamlı sonuçlar elde ettik. Bu durum tip ॥ DM' un mikrovasküler komlikasyonlarından olan DR ile yüksek HbA1c düzeyleri arasında önemli ilişki olduğunu göstermektedir.

Bu yapılan çalışma da görülmektedir ki tip II DM hastası olup DR gelişen hastalarda HbA1c düzeylerinde önemli derecede artış vardır. HbAlc düzeyleri arttıkça DR görülme sıklığı da artmaktadır. Ayrıca DR uzun süre bu hastalığa maruz kalan hastalarda görüldüğünden tip II DM hastaların takibinde ve komplikasyonların önlenmesinde kan glukoz regülasyonunun sağlanması ve HbA1c düzeylerini normal sınırlarda tutmanın önemi büyüktür.

\section{KAYNAKLAR}

1. Satman i. The update criteria and the reasons of them in diagnosis and follow up of diabetes mellitus. Turkiye Klinikleri J Int Med Sci. 2007;3:1-15.

2. Yıldırım Di, Marakoğlu K. Diyabet Hastalarında D Vitamini ile Hba1c illişkisinin Değerlendirilmesi. Selcuk Med J. 2019;35(1):37-42.

3. Ali A, Iqbal F, Taj A, Iqbal Z, Amin MJ, Iqbal QZ. Prevalence of microvascular complications in newly diagnosed patients with type 2 diabetes. Pak J Med Sci. 2013;29(4):899-902. 
4. Hayrullah Y, Mehmet B, Mustafa KB, Goçmen AY. The effects of dialysers on some blood biochemical parameters in hemodialysis patients. A J P P Sci. 2011;5(22):2513-2516. doi: 10.5897/AJPP 11.690.

5. Abdülhekim $Y$, Hayrullah $Y$, Mehmet A, Ahmet $P$, Suleyman K. Refractive errors in patients with newly diagnosed diabetes mellitus. Pak J Med Sci. 2015;31(6):1481-1484.

6. American Diabetes Association (ADA). Standards of Medical Care in Diabetes. Diabetes Care, 2015;29(1):43-48

7. Türkiye Endokrinoloji ve Metabolizma Derneği (TEMD). Diabetes Mellitus ve Komplikasyonlarının Tanı, Tedavi Ve İzlem Kılavuzu, 2016. http://www.turkendokrin.org/files/file/DIYABET_TTK_web.pdf (Erişim Tarihi:04.02.2017)

8. Özcan, Ş. Kronik Komplikasyonlar, (Eds: Erdoğan S.), Diyabet Hemşireliği Temel Bilgiler, Yüce Basımevi, İstanbul, 2002;141-156.

9. Taş A, Bayraktar M.Z, Erdem Ü, Sobacı G, Uçar M. Diyabetik hastalarda retinopati sıklığı ve risk faktörleri. Gülhane Tıp Dergisi, 2005;47:164-174.

10. Özmen B, Güçlü F, Kafesçiler S, Özmen D, Hekimsoy Z. The relationship between glycosylated haemoglobin and diabetic retinopathy in patients with type 2 diabetes.department of endocrinology. Turkish Journal Endocrinology, 2007;11:10-15.

11. Mehmet Ö, Hayrullah Y, Durhasan M. HbA1c Testi İçin Maliyet Etkinlik Değerlendirmesi. Adıyaman Üniversitesi. Sağlık Bilimleri Dergisi. 2019; 5(1);1335-1341.

12. Ferrer EJ, Licea Puig M, Diaz Diaz O. Giycosylated hemoglobin as a prognostic index of the appearance of vascular comlications in diabetic patients. Rev Clin Esp. 1990;187(3):121-4.

13. Agardh E, Agardh CD, Koul S, Torffvit O. A four-year follow- up study on the incidence of diabetic retinopathy in older onset diabetes mellitus|published erratum appears in Diabet Med 1994 Jul; 11(6):5241. Diabet Med.11(3):273-8.

14. Diabet komplikasyon istatistikleri. Hatemi H. İstanbul.1996.

15. American Diabetes Association. Standarts of Medical Care in Diabetes-2018. "6. Glycemic Targets: Standards of MedicalCare in Diabetes-2018" Diabetes Care. 2018;41(Suppl 1):55-64.

16. Park CY, Park SE, Bae JC. Prevalence of and risk factors for diabetic retinopathy in Koreans with type II diabetes: baseline characteristics of Seoul Metropolitan City-Diabetes Prevention Program (SMC-DPP) participants. Br J Ophthalmol. 2012;96:151-5.

17. Kim JH, Kwon HS, Park YM. Prevalence and associated factors of diabetic retinopathy in rural Korea: the Chungju metabolic disease cohort study. J Korean Med Sci. 2011;26:1068-73.

18. Xu J, Wei WB, Yuan MX, Wan g, Zheng YY, Li YB et al. Prevalence and risk factors for diabetic retinopathy: the Beijing Communities Diabetes Study 6. Retina. 2012;32:322-9. 